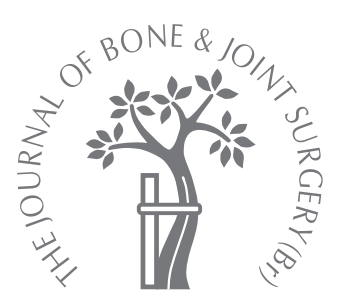

J.-M. Brinkman,

P. J. A. Schwering,

L. Blankevoort,

J. G. Koolos,

J. Luites,

A. B. Wymenga

From St.

Maartenskliniek,

Nijmegen, The

Netherlands

J.-M. Brinkman, MD

Orthopaedic Resident

J. Luites, Researcher

A. B. Wymenga, MD, PhD,

Orthopaedic Surgeon

Department of Orthopaedics

St. Maartenskliniek, P. O. Box

9011, 6500 GM Nijmegen,

The Netherlands.

P. J. A. Schwering, MD, Orthopaedic Surgeon

Department of Orthopaedics Canisius-Wilhelmina

Hospital, P. O. Box 9015, 6500

GS, Nijmegen, The

Netherlands.

L. Blankevoort, PhD,

Engineer

Orthotrauma Research

Center

Academic Medical Center,

P. O. Box 22660, 1100 DD,

University of Amsterdam,

Amsterdam, The

Netherlands.

J. G. Koolos, PhD,

Anatomist

Department of Anatomy and

Embryology

University of Nijmegen, P. O.

Box 9101, $6500 \mathrm{HB}$,

Nijmegen, The Netherlands.

Correspondence should be sent to Dr A. B. Wymenga:

e-mail: a.wymenga@

maartenskliniek.nl

(C)2005 British Editorial Society of Bone and Joint Surgery

doi:10.1302/0301-620X.87B10. $16536 \$ 2.00$

$J$ Bone Joint Surg [Br] 2005;87-B:1364-8.

Received 24 March 2005;

Accepted 10 May 2005

\title{
The insertion geometry of the posterolateral corner of the knee
}

\begin{abstract}
We have quantitatively documented the insertion geometry of the main stabilising structures of the posterolateral corner of the knee in $\mathbf{3 4}$ human cadavers. The lateral collateral ligament inserted posterior $(4.6 \mathrm{~mm}, \mathrm{SD} 2)$ and proximal $(1.3 \mathrm{~mm}, \mathrm{SD} 3.6)$ to the lateral epicondyle of the femur and posterior $(8.1 \mathrm{~mm}, \mathrm{SD} 3.2)$ to the anterior point of the head of the fibula. On the femur, the popliteus tendon inserted distally $(11 \mathrm{~mm}$, SD 0.8$)$ and either anterior or posterior (mean $0.84 \mathrm{~mm}$ anterior, SD 4) to the lateral collateral ligament. The popliteofibular ligament inserted distal $(1.3 \mathrm{~mm}, \mathrm{SD} 1.2)$ and anterior $(0.5 \mathrm{~mm}, \mathrm{SD} 2.0)$ to the tip of the styloid process of the fibula.

The ligaments had a consistent pattern of insertion and, despite the variation between specimens, the standard deviations were less than the typical size of drill hole used in reconstruction of the posterolateral corner. The data provided in this study can be used in the anatomical repair and reconstruction of this region of the knee.
\end{abstract}

In recent years it has become increasingly clear that the posterolateral corner of the knee plays an important role in maintaining stability. ${ }^{1}$ The structures of the posterolateral corner play a major role in resisting varus and external rotation forces and posterior translation. ${ }^{2-5}$ Isolated lesions of the posterolateral corner are rare but can nevertheless cause severe disability because of instability and secondary degeneration of the articular cartilage. ${ }^{6}$ Most injuries to the area occur in combination with disruption of the posterior cruciate ligament (PCL) or, to a lesser extent, the anterior cruciate ligament (ACL). ${ }^{6,7}$ It has been suggested that unrecognised and subsequently untreated injury to the posterolateral corner is associated with an increased risk of failure of a reconstructed cruciate ligament. ${ }^{8-10}$ The goals of surgical repair are to restore normal knee kinematics and to reduce the risk of progressive degenerative arthritis. ${ }^{11}$ However, the posterolateral corner has a complex and variable anatomy and there is no current consensus on the operative treatment of choice, although various techniques of isometric and anatomical reconstruction have been developed. ${ }^{1,11-15}$ Anatomical reconstruction requires exact knowledge of the insertion geometry of the structures involved. The lateral collateral ligament (LCL), the popliteus complex (popliteus and its tendon), and the popliteofibular ligament, are considered to be the main contributors to posterolateral stability. ${ }^{2-4,16-20}$
Their functional contribution has been welldocumented in a number of studies. ${ }^{2,3,8,20,21}$ Reconstruction of the corner requires the ability to identify anatomical landmarks peri-operatively. Geometric data to assist with this have only once been published, by LaPrade et al. ${ }^{16}$ Consequently, the aim of this cadaver dissection study was to provide data, which could be used peri-operatively, on the insertion geometry of the LCL, the popliteus tendon and the popliteofibular ligament and their variability in the human knee, by reference to relevant anatomical and surgical landmarks.

\section{Materials and Methods}

We dissected 34 intact human, cadaver, formalin-preserved knee joints from 34 adults without signs of gross bony deformity, previous fracture or degenerative disease and with intact ligaments in the posterolateral corner. No data on the age and gender of the donors were available, but usually they were older than 60 years. The LCL, the popliteus muscle and tendon and the popliteofibular ligament were identified and the circumference of their insertion into the femur and the fibula marked with lead wires. Relevant bony landmarks on the femur and head of the fibula were similarly marked. On the femur these landmarks were the lateral epicondyle and the lateral cartilaginous border of the lateral condyle (Fig. 1). On the head of the fibula two landmarks were chosen; the 


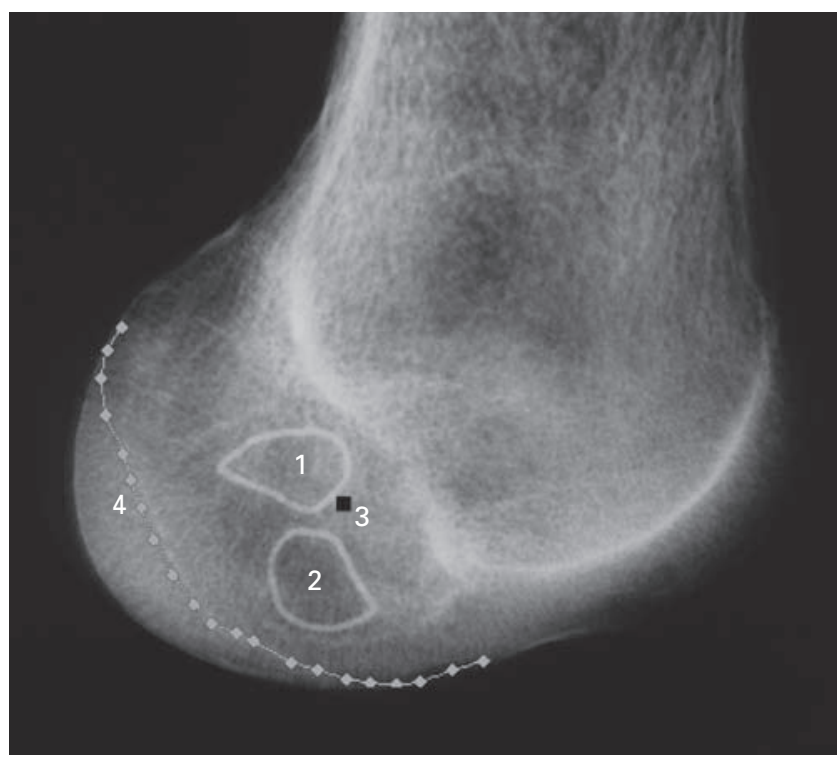

Fig. 1

Lateral radiograph showing the insertions of 1 , the lateral collateral ligament; 2 , popliteus tendon; 3 , the epicondyle; and 4, the lateral cartilaginous border.

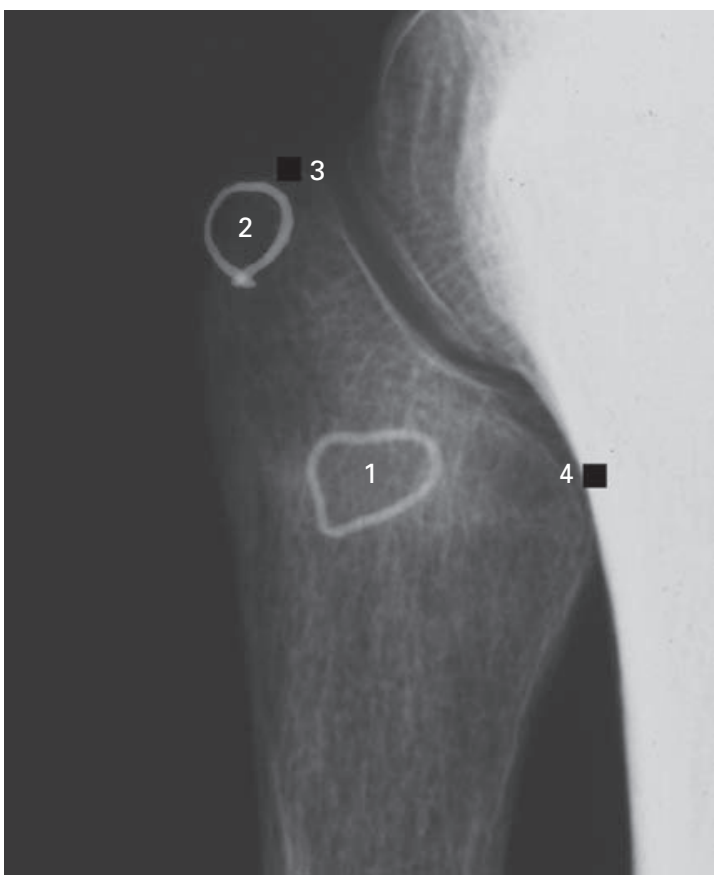

Fig. 2

Lateral radiograph of a tibiofibular combination showing the insertions of 1 , the lateral collateral ligament; 2 , popliteofibular ligament; 3 , the proximal point; and 4, the anterior point.

most proximal point on the styloid process and the most anterior point on the anterior surface of the head (Fig. 2). After dissection and marking, each femur and each tibiofibular combination was embedded in polymethylmeth- acrylate cement in a box-shaped positioning and cementing device, which allowed for placement in the anatomical position as described by Staubli, Noesberger and Jakob. ${ }^{22}$ The posterior aspect of the femoral and tibial condyles were placed against a supporting plate so that the posterior condylar plane and the tibial plateau, respectively, were parallel to the base of the model. The femur and tibia were placed against a second supporting plate so that in the sagittal plane the posterior femoral and tibial cortices were perpendicular to the base of the model. Once embedded in this position, the posterior condyles projected onto each other on plain lateral radiographs.

To measure the three-dimensional (3D) position of the marked insertions and landmarks a 3D measuring system (3 Space Isotrak, Polhemus Navigation Sciences, Colchester, Vermont) was used. This is an electromagnetic device comprising of a source and a sensor, which is capable of measuring and recording the position in space of the tip of a stylus connected to the sensor, to an accuracy of $0.35 \mathrm{~mm}$. The tip of the stylus is first used to calibrate the measuring system and define the axis, using specific points on the positioning and cementing device. Thereafter, the outlines of the insertions are traced using the stylus and their position is recorded and converted into $\mathrm{x}, \mathrm{y}$ and $\mathrm{z}$ coordinates. In order to standardise all geometrical data, measurements for each femur and corresponding tibiofibular combination were scaled linearly up or down to the mediolateral transepicondylar width $(80 \mathrm{~mm})$ of a standard-size femur as described by Walker et $\mathrm{al}^{23}$ and Mensch and Amstutz. ${ }^{24}$ Thereafter, using the coordinates of the anatomical landmarks and insertions, a graphic model was created for each femur and fibula and the geometric centre of each insertion was calculated. The position of the popliteus tendon and LCL insertion was formed by calculating the distance from the central point of each insertion to the lateral epicondyle and their distance relative to one other. Similarly, in the fibular graphic model the positions of the popliteofibular ligament and LCL insertions were determined by calculating the distance from the centre of each insertion to the proximal and anterior point. Again, the position of the insertions relative to one other was also calculated. Finally, for each insertion, and again using the co-ordinates recorded with the Isotrak system (3 Space Isotrak), the surface area of the insertion was calculated. This was the area of the best-fit plane through the measurement points, within the circumference defined by the points.

\section{Results}

In all 34 knees, the LCL, the popliteus muscle and tendon and the popliteofibular ligaments were well-defined structures (Fig. 3). Using the recorded data and the graphic model of each femur and fibula, the position of the LCL, and insertions of the popliteus tendon and popliteofibular ligament were calculated relative to the bony landmarks. All calculations and measurements refer to the central point of ligament insertion. 


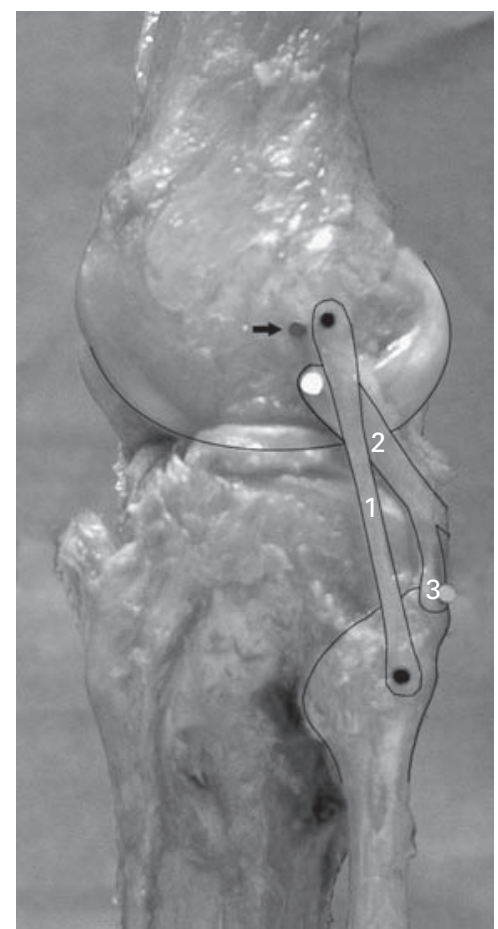

Fig. 3a

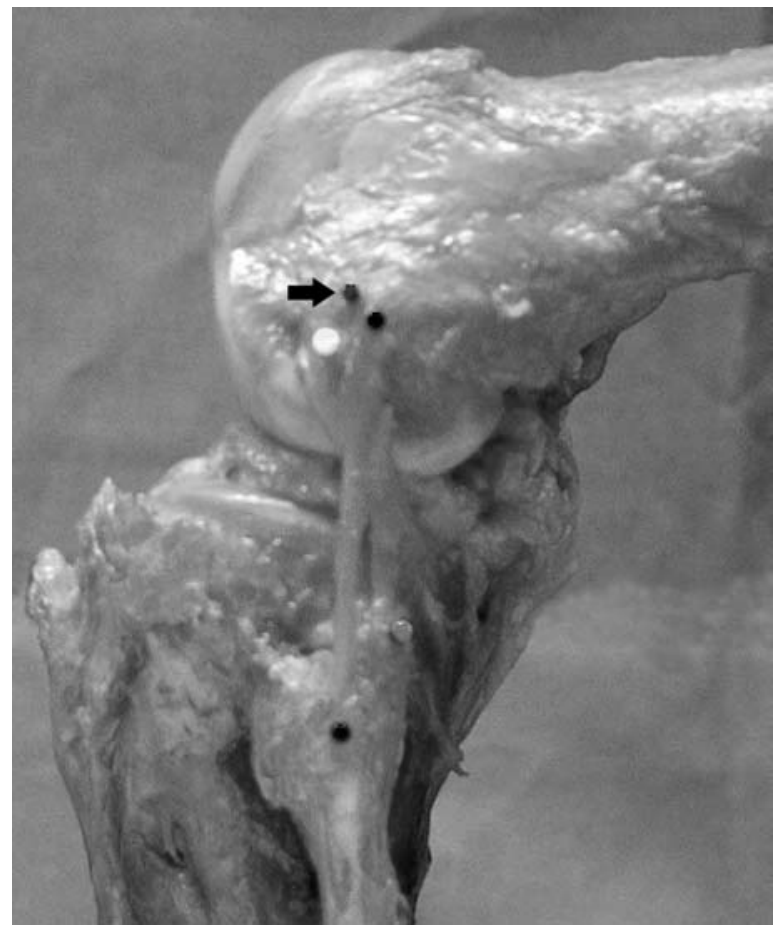

Fig. 3b

Figure 3a - A knee specimen in extension; the lateral epicondyle (arrow); 1, lateral collateral ligament (LCL); 2 , popliteus tendon; and 3, popliteofibular ligament are shown. The insertion of the popliteus tendon onto the femur is posterior to the epicondyle, and anterior to the LCL insertion. Figure $3 b-T h e$ same knee in flexion; the insertion of the popliteus tendon (white marker) is now located anterior to the lateral epicondyle (arrow), and the angle between the insertions of the LCL and popliteus tendon has increased.

Femur. The LCL inserted a mean of $1.3 \mathrm{~mm}$ (SD 3.6, -6.3 to 7.8 ) proximal and $4.6 \mathrm{~mm}$ (SD 2.0, -1.0 to 8.1 ) posterior to the lateral epicondyle; 23 insertions were located proximal and 11 distal to the epicondyle, 32 were located posterior and two anterior. The popliteus tendon inserted a mean of $9.7 \mathrm{~mm}$ (SD 3.9, 1.0 to 17.3 ) distal and $5.3 \mathrm{~mm}$ (SD 5.4, 1.5 to 13.4 ) posterior to the lateral epicondyle. All insertions of the popliteus tendon were located distally and posteriorly to the lateral epicondyle, except one, which was proximal and two which were anterior. The popliteus tendon inserted a mean of $11 \mathrm{~mm}$ (SD 4.0, 3.5 to 17.9 ) distal and $0.84 \mathrm{~mm}$ (SD $4,-8.5$ to 9.5 ) anterior to the LCL insertion. Nineteen insertions of the popliteus tendon were located anterior and 15 posterior to the LCL insertion. The variation in the location of the insertions of the LCL and popliteus tendon on the femur is shown in Figure 4. The mean surface area of the insertions was $51.7 \mathrm{~mm}^{2}$ (22.9 to 88.8) for the LCL and $65.9 \mathrm{~mm}^{2}$ (31 to 104 ) for the popliteus tendon, respectively. Fibula. All insertions of the popliteofibular ligament were located on the styloid process of the head of the fibula; the central point of each insertion was located immediately anterior (22 insertions) or posterior (12) to the most proximal point. Insertions were located a mean of $1.3 \mathrm{~mm}$ distally (SD 1.2, 0.6 to 3.9) and a mean of $0.50 \mathrm{~mm}$ (SD 2.0, -3.5 to 4.4 ) anteriorly. The LCL inserted $13.7 \mathrm{~mm}$ (SD 2.9, 7.6 to 19.2 ) distal and $11.7 \mathrm{~mm}$ (SD $4.5,4.0$ to 21.6 ) ante- rior to the most proximal point on the styloid process. The LCL insertion was a mean of $11.2 \mathrm{~mm}$ (SD $4.6 ;-1.3$ to 20.8 ) anterior and $12.3 \mathrm{~mm}$ (SD $3.3 ; 2.5$ to 20.3 ) distal to the insertion of the popliteofibular ligament. All LCL insertions were located anterior to the insertion of the popliteofibular ligament and anterior to the most proximal point on the head of the fibula (Fig. 5). The LCL insertion was a mean of $8.1 \mathrm{~mm}$ (SD 3.2, 2.0 to 16.1) posterior and $0.40 \mathrm{~mm}$ (SD 3.5, -5.9 to 7.2 ) distal relative to the most anterior point of the head; 16 insertions were located proximally and 19 distally. The mean surface area of the insertions was $35 \mathrm{~mm}^{2}$ (15 to 84 ) for the LCL and $17 \mathrm{~mm}^{2}$ (8.6 to 33.4 ) for the popliteofibular ligament, respectively.

\section{Discussion}

The aim of this study was to provide data on the insertion geometry of the major structures of the posterolateral corner by using identifiable bony landmarks. We found that on the femur and fibula the insertion sites of the LCL, popliteus tendon and popliteofibular ligament can be described in a reproducible way relative to the lateral epicondyle of the femur, and the styloid process and anterior border of the head of the fibula. The position of the insertions was largely similar between the 34 specimens and in agreement with previous reports. ${ }^{14,16-18,20}$ However, the data show that there is some variation in the position of the insertions 


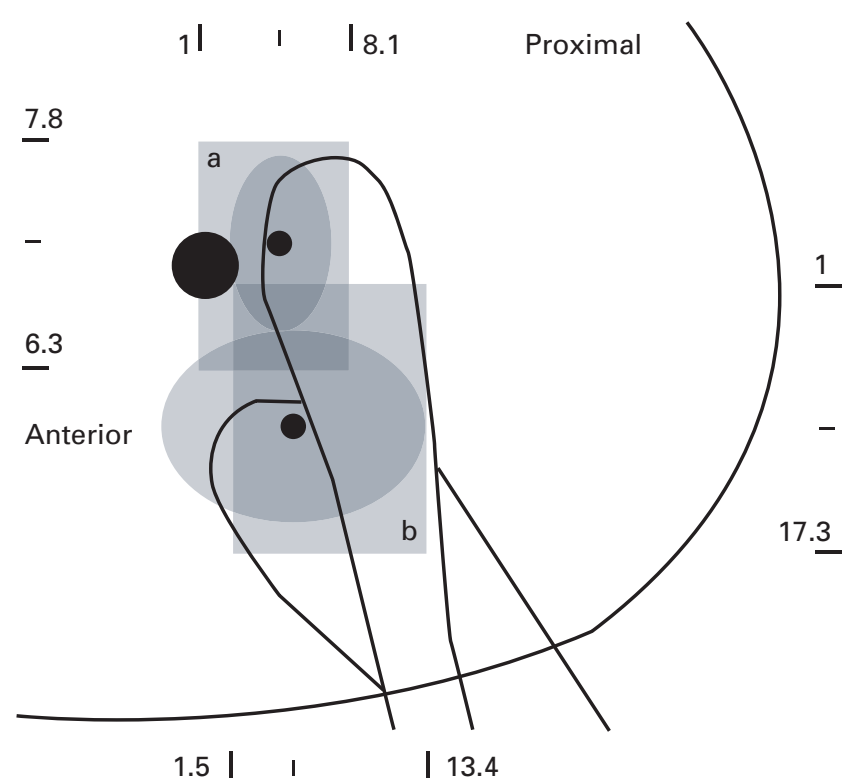

Fig. 4

Schematic overview of the insertions of the lateral collateral ligament (LCL) and popliteus tendon on the femur, showing the range of the insertions (light grey) of the LCL (a) and popliteus tendon (b) and twice the standard deviation (dark grey). All measurements are in $\mathrm{mm}$.

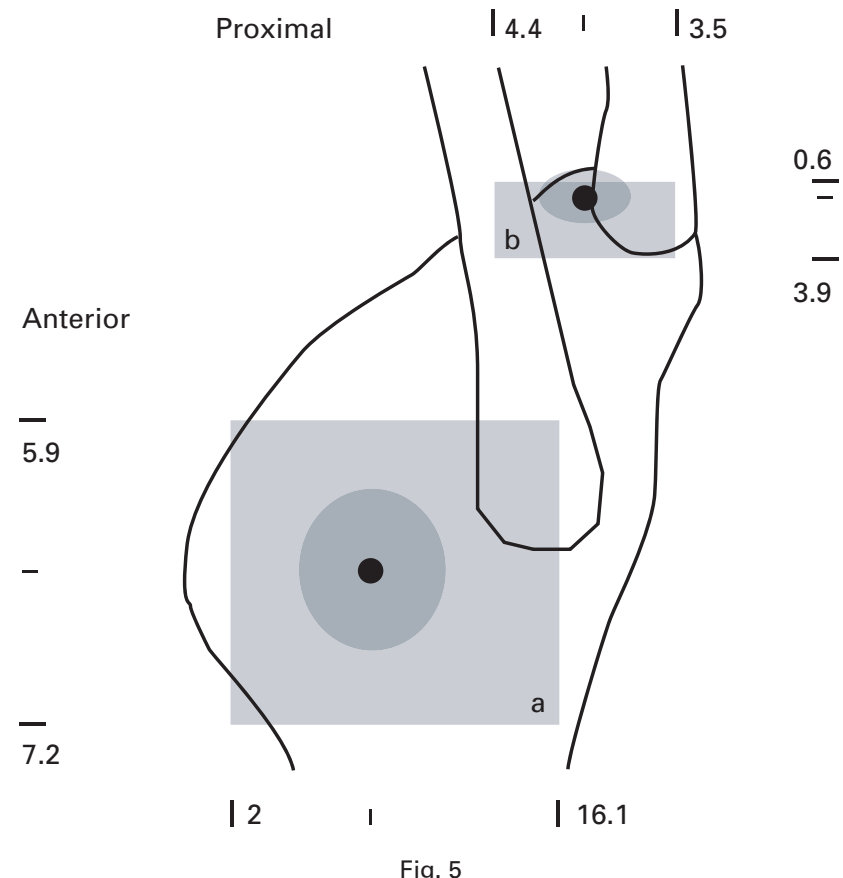

Schematic overview of the insertions of the lateral collateral ligament (LCL) and popliteofibular ligament on the fibula, showing the range of the insertions (light grey) of the LCL (a) and popliteofibular ligament (b) twice the standard deviation (dark grey). All measurements are in $\mathrm{mm}$.

in the posterolateral corner between specimens, most notably for the insertion of the LCL and popliteus tendon on the femur. In contrast with earlier reports, our study provides both quantitative and qualitative information about the posterolateral corner, and the insertion of popliteus onto the fibula.

LaPrade et $\mathrm{al}^{16}$ gave a qualitative and quantitative morphological analysis of the LCL, popliteus tendon, popliteofibular ligament and lateral gastrocnemius tendon in ten fresh-frozen cadaver knees. We agree with their findings as our specimens demonstrated that the LCL had no significant attachment to the lateral epicondyle itself. The LCL inserted a mean of $4.6 \mathrm{~mm}$ posteriorly and $1.3 \mathrm{~mm}$ proximally to the lateral epicondyle, similar to the $3.1 \mathrm{~mm}$ and $1.4 \mathrm{~mm}$, respectively, documented by LaPrade et al. ${ }^{16}$ Our data also showed a greater variation in the insertion site of the LCL, which was not always located proximal and posterior to the epicondyle, as reported by LaPrade et al. ${ }^{16}$ From a practical standpoint, the remains of the LCL insertion can usually be found during surgery, except when the epicondylar area has been fractured or if previous surgery has been performed.

We found that the popliteus tendon generally inserted in a straight line, parallel to the long axis of the femur, a mean of $11 \mathrm{~mm}$ distal and $0.84 \mathrm{~mm}$ anterior to the LCL insertion. However, this position varied between $8.5 \mathrm{~mm}$ posterior and $9.5 \mathrm{~mm}$ anterior to the LCL. Quantitatively, LaPrade et $\mathrm{al}^{16}$ reported that the popliteus tendon invariably inserted anterior to the LCL insertion, positioned $18.5 \mathrm{~mm}$ obliquely to it, in the proximal half and anterior one-fifth of the popliteal sulcus. Qualitatively, LaPrade et $\mathrm{al}^{16}$ and Staubli and Birrer ${ }^{18}$ described the popliteus tendon as inserting proximally, at the anterior end of the popliteal sulcus on the lateral femoral condyle. The popliteal sulcus is a reliable landmark which can be used as a peri-operative reference point and is usually easily palpable. However, we did not document its position in our study.

The position of the insertions on the head of the fibula is similar to data reported by others. ${ }^{16,18}$ Invariably, all insertions of the popliteofibular ligaments were closely related to the tip of the styloid process. The LCL inserted immediately above, or below, a straight line through the anterior point of the head, a mean of $8.2 \mathrm{~mm}$ posteriorly and $0.40 \mathrm{~mm}$ distally, compared with the $8.2 \mathrm{~mm}$ measured by LaPrade et al. ${ }^{16}$ During surgery, the remains of the LCL insertion onto the fibula can usually be found below the bursa of the long head of the biceps.

Apart from the variation in the position of the insertions of the LCL and popliteus tendon, we also found a large variation in the calculated surface area of the insertions for both from 31 to $104 \mathrm{~mm}^{2}$ for the popliteus tendon and from 15 to 84 $\mathrm{mm}^{2}$ for the LCL. These figures compared with 53 to $62 \mathrm{~mm}^{2}$ and 62 to $73 \mathrm{~mm}^{2}$, respectively, reported by LaPrade et al. ${ }^{16}$

The standard deviations for the insertion locations on the femur and fibula in our study are relatively small, ranging from 1.2 to $5.4 \mathrm{~mm}$, particularly when compared with the diameter $(8 \mathrm{~mm})$ of a typical drill hole used in reconstruction of the posterolateral corner. This suggests that there will always be an overlap in its position if mean values for insertion geometry are used. 
Whether differences between our data and that provided by LaPrade et $\mathrm{a}^{16}$ are because of the larger number of knees we used, and therefore a reflection of normal variation, or perhaps differences in measurement technique and study design, cannot be determined. A direct comparison of the measurements between LaPrade et $\mathrm{al}^{16}$ and our study may not be reliable. Except for the LCL insertion onto the femur, LaPrade et $\mathrm{al}^{16}$ only reported the direct distance between the insertions of the LCL and the popliteus tendon $(18.5 \mathrm{~mm})$ on the femur and between the anterior point of the head and the LCL on the fibula $(8.2 \mathrm{~mm})$. For our study those values were $11 \mathrm{~mm}$ and $8.2 \mathrm{~mm}$, respectively. LaPrade et $\mathrm{al}^{16}$ did not provide data on the position of these structures in either craniocaudal or anteroposterior planes and did not report on how specimen positioning was controlled or if scaling was performed. Furthermore, a difference in the angle between the femur and tibia/fibula during measurements may account for part of the differences in the position of the insertion of the popliteus tendon relative to the insertion of the LCL (Fig. 3). In our study the angle between the femur and tibia/fibula was $0^{\circ}$, because both the posterofemoral and tibial cortices were parallel.

This dissection study of the posterolateral corner has provided further quantitative data on the insertion geometry of the LCL, popliteus tendon and popliteofibular ligament. The insertions of the LCL and popliteus tendon onto the femur and fibula can be described in a reproducible way. We consider that these data can be safely used in the anatomical repair and reconstruction of the posterolateral structures of the knee.

No benefits in any form have been received or will be received from a commercial party related directly or indirectly to the subject of this article.

\section{References}

1. Davies H, Unwin A, Aichroth P. The posterolateral corner of the knee: anatomy, biomechanics and management of injuries. Injury 2004;35:68-75.

2. Gollehon DL, Torzilli PA, Warren RF. The role of the posterolateral and cruciate ligaments in the stability of the human knee: a biomechanical study. J Bone Joint Surg [Am] 1987;69-A:233-42

3. Grood ES, Stowers SF, Noyes FR. Limits of movement in the human knee: effect of sectioning the posterior cruciate ligament and posterolateral structures. J Bone Joint Surg $[\mathrm{Am}] 1988 ; 70-\mathrm{A}: 88-97$
4. Veltri DM, Deng XH, Torzilli PA, Maynard MJ, Warren RF. The role of the popliteofibular ligament in stability of the human knee: a biomechanical study. Am J Sports Med 1996;24:19-27.

5. Markolf KL, Wascher DC, Finerman GA. Direct in vitro measurement of forces in the cruciate ligaments. Part II: the effect of section of the posterolateral structures. $J$ Bone Joint Surg [Am] 1993;75-A:387-94.

6. DeLee JC, Riley MB, Rockwood CA Jr. Acute posterolateral rotatory instability of the knee. Am J Sports Med 1983;11:199-207.

7. Hughston JC, Jacobson KE. Chronic posterolateral rotatory instability of the knee. $J$ Bone Joint Surg [Am] 1985;67-A:351-9.

8. Harner CD, Vogrin TM, Hoher J, Ma CB, Woo SL. Biomechanical analysis of a posterior cruciate ligament reconstruction: deficiency of the posterolateral structures as a cause of graft failure. Am J Sports Med 2000;28:32-9.

9. LaPrade RF, Muench C, Wentorf F, Lewis JL. The effect of injury to the posterolateral structures of the knee on force in a posterior cruciate ligament graft: a biomechanical study. Am J Sports Med 2002;30:233-8.

10. Noyes FR, Barber-Westin SD, Roberts CS. Use of allografts after failed treatment of rupture of the anterior cruciate ligament. J Bone Joint Surg [Am] 1994;76-A: 1019-31.

11. Covey DC. Injuries of the posterolateral corner of the knee. J Bone Joint Surg [Am] 2001:83-A:106-18.

12. Fanelli GC, Larson RV. Practical management of posterolateral instability of the knee. Arthroscopy 2002;18(2 Suppl 1):1-8.

13. Larson RV. Isometry of the lateral collateral and popliteofibular ligaments and techniques for reconstruction using a free semitendinosus graft. Oper Tech Sports Med 2001;9:84-90.

14. Terry GC, LaPrade RF. The posterolateral aspect of the knee: anatomy and surgical approach. Am J Sports Med 1996;24:732-9.

15. Veltri DM, Warren RF. Operative treatment of posterolateral instability of the knee. Clin Sports Med 1994;13:615-27.

16. LaPrade RF, Ly TV, Wentorf FA, Engebretsen I. The posterolateral attachments of the knee: a qualitative and quantitative morphologic analysis of the fibular collateral ligament, popliteus tendon, popliteofibular ligament, and lateral gastrocnemius tendon. Am J Sports Med 2003;31:854-60.

17. Seebacher JR, Inglis AE, Marshall JL, Warren RF. The structure of the posterolateral aspect of the knee. J Bone Joint Surg [Am] 1982;64-A:536-41.

18. Staubli HU, Birrer S. The popliteus tendon and its fascicles at the popliteal hiatus: gross anatomy and functional arthroscopic evaluation with and without anterior cruciate ligament deficiency. Arthroscopy 1990;6:209-20.

19. Veltri DM, Warren RF. Anatomy, biomechanics, and physical findings in posterolateral knee instability. Clin Sports Med 1994;13:599-614.

20. Watanabe $\mathbf{Y}$, Moriya $\mathbf{H}$, Takahashi $\mathbf{K}$, et al. Functional anatomy of the posterolateral structures of the knee. Arthroscopy 1993;9:57-62.

21. Vogrin TM, Hoher J, Aroen A, Woo SL, Harner CD. Effects of sectioning the posterolateral structures on knee kinematics and in situ forces in the posterior cruciate ligament. Knee Surg Sports Traumatol Arthrosc 2000;8:93-8.

22. Staubli HU, Noesberger B, Jakob RP. Stress radiography of the knee: cruciate ligament function studied in 138 patients. Acta Orthop Scand Supp/ 1992;249:1-27.

23. Walker PS, Kurosawa H, Rovick JS, Zimmerman RA. External knee joint design based on normal motion. J Rehabil Res Dev 1985;22:9-22.

24. Mensch JS, Amstutz HC. Knee morphology as a guide to knee replacement. Clin Orthop 1975;112:231-41. 